\title{
Evaluation of Diagnostic Hysteroscopy as a Tool for Diagnosis of Tubal Factor of Infertility in Comparison to Diagnostic Laparoscopy
}

\author{
Mahmoud El Zoghby Abdel Rahman Hefny ${ }^{1, *}$ MSc, Mohammad Salah El Din Hassanin ${ }^{1}$ MD, \\ Bassem Ragab Abdel Aziz ${ }^{1}$ MD.
}

\section{*Corresponding Author: \\ Mahmoud El Zoghby Abdel Rahman Hefny e7odaa@gmail.com}

Received for publication August 14, 2020; Accepted October 12, 2020;

Published online October 12, 2020.

Copyright 2020 The Authors published by Al-Azhar University, Faculty of Medicine, Cairo, Egypt. All rights reserved. This an openaccess article distributed under the legal terms, where it is permissible to download and share the work provided it is properly cited. The work cannot be changed in anyway or used commercially.

doi: 10.21608/aimj.2020.39083.1305

${ }^{1}$ Obstetrics and Gynecology Department, Faculty of Medicine, Al-Azhar University,Cairo,Egypt.

\begin{abstract}
Background: Fallopian tube obstruction is believed to play a role in 12$33 \%$ of subfertile couples. Therefore, the patency evaluation of fallopian tubes is a significant part of subfertile couple's work.

Aim of the work: This work aims at comparing the sensitivity and specificity of the hysteroscopic assessment of tubal patency by the bubble test to diagnostic laparoscopy and hysterosalpingogram in infertile patients.

Patients and Methods: A total of 100 women were recruited to the study during the period between January 2020 and June 2020. 100 patients underwent a diagnostic hysteroscopy followed by laparoscopy. Each Fallopian tube has been considered to be an individual case. Patency was evaluated in 200 Fallopian tubes.

Results: A polyp was detected at three tubal orifice. Hyperemia was detected around two tubal orifice. Adhesions were detected at one tubal orifice. The tubes were patent in 113 cases with both methods. In 70 cases both laparoscopy and hysteroscopy found occluded tubes. The tubes were patent by laparoscopic dye in 10 cases, but could not be detected by hysteroscopy as such. Occlusion was observed by laparoscopy in 7 cases but patency was detected during hysteroscopy. Of the non-patent cases, 70 out of 77 laparoscopic findings were consistent with the hysteroscopic result, yielding a specificity estimate of $90.9 \%$ (95\% CI 84.5\% to 96.4\%).

Conclusion: Based on statistical analysis (sensitivity and specificity) of our data, diagnostic hysteroscopic bubble test is accurate and reliable compared to the laparoscopic test, which is considered a global standard criterion.
\end{abstract}

Keywords: Transvaginal Ultrasound; Hysteroscopy; Dilatation and Curettage

Disclosure: The authors have no financial interest to declare in relation to the content of this article. The Article Processing Charge was paid for by the authors.

Authorship: All authors have a substantial contribution to the article.

\section{INTRODUCTION}

TVS-associated diagnostic hysteroscopy can be used as an alternative to HSG, as an efficient invasive diagnostic technique that can be used in combination with tubes in infertile women as a method for uterine cavity evaluation. ${ }^{1}$

Diagnostic hysteroscopy was the preferred method for assessing the cervical canal and uterine cavity. Since the scopes and instruments are miniaturized. ${ }^{5}$

No single method accurately identified all the various pathologies, with hysteroscopy being the gold standard for the detection of cervical and endometrial pathologies, adding to it the detection of tubal 
HSG, HSC and TVS may be chosen on the basis of patient clinical presentation.$^{13}$

A new modality hystero-contrast sonography (HyCoSy) showed good statistical comparability and consistency with HSG, combined with dye laparoscopy. HyCoSy is tolerated well and can be an appropriate alternative outpatient procedure. HyCoSy with contrast agent appears more effective in detecting tubal obstruction than saline solution.$^{11}$

The most popular cause for infertility is the obstruction of the Fallopian tubes, checking tubal patency is the first line of fertility check-up so that, the release of air bubbles during hysteroscopy is an indirect indication of tubal patency ${ }^{9}$

\section{PATIENTS AND METHODS}

This is a cross sectional cohort research at the Department of Obstetrics and Gynecology at the University of Al Azhar (Bab El Shaaria and El Hussein University hospitals) within a period of 6 months (January 2020 and June 2020). The protocol of this study was approved by the department \& college research ethics committees. The patients were counselled and written informed consent was obtained before inclusion in the study. One hundred infertile women referred for diagnostic/operative laparoscopy was included in the study.

The patients included underwent full history and physical examination. All patients used to have hormonal profile, fundamental transvaginal ultrasonography (TVS), semen examination, and hysterosalpingography (HSG) in the form of infertility work-up.

Inclusion Selection of patients was based on: Female patients 20 to 40 years of age. Primary or secondary infertil patients. All examination will be performed between the 5th and the 10th cycle days.

Exclusion criteria: Concomitant neurologic disease which could affect the correct pain assessment. Pregnancy. Any hysteroscopic contraindication such as Pelvic inflammatory disease (PID). Severe bleeding in the uterus. Any uterine abnormality such as pinhole cervix which would prevent a catheter from passing through the cervix.

Diagnostic hysteroscopy was performed by hysteroscopists who were blind to the HSG results and laparoscopy was performed by laparoscopists who were blind to the results of diagnostic hysteroscopy and HSG.

Diagnostic hysteroscopy was performed during the proliferative phase of the menstrual cycle. We used a rigid 2.9-mm hysteroscope with an oblique lens $30^{\circ}$ forward and $5 \mathrm{~mm}$ outer sheath diameter (Karl Storz GmbH, Tuttlingen, Germany) in this study . ${ }^{5}$ The uterine cavity was distended using normal saline solution. The distension media pressure was maintained at 60 to $100 \mathrm{~mm} \mathrm{Hg}$. Hysteroscopy was conducted using a non-touch (vaginoscopic approach) technique described by Evangelista .
The uterine cavity was investigated systematically, beginning with its anterior and posterior walls, the fundus, and the borders. The size and equality of the tubal orifices were observed and any pathology found at the tubal orifices (adhesions, polyps or hyperemia or inflammatory changes) were reported. Any air bubbles in the irrigating fluid were reported to pass to tubal ostea. If no air bubbles were noticed, we just injected $2 \mathrm{ml}$ of air into the sterile infusion rubber end set for the hysteroscopist to see. Hysteroscopic suction test for bubble was considered positive if there was an air bubble suction of ostium on the patent side within 1 min. Neither air injection nor increased pressure during this period. When gas bubbles didn't suck, the examiner waited another 1 min. Again, if no suction was taken, the test was deemed negative ${ }^{3}$

Laparoscopy was performed under general anesthesia using a 10-mm scope (Karl Storz, Tuttlingen, Germany) and two ancillary $5 \mathrm{~mm}$ trocars in the lower abdomen.The cervix was dilated to Hegar 8 and a uterine manipulator (Cooper Surgical Rumi) was placed (Fig. 5). The uterine manipulator was connected to a syringe containing $20 \mathrm{ml}$ of saline with a blue methylene dye. The uterus, tubes, ovaries and neighbouring organs were thoroughly inspected and the presence of any abnormality such as endometriosis, fibroids or adhesions was noted.The assistant injected the methylene blue dye and the spill of the dye through the fallopian tubes was observed. Suction and irrigation cannula was inserted in the supra pubic port for suction of the methylene blue dye out of the peritoneal cavity. Gas was then evacuated from the peritoneal cavity and the sites of the ports were sutured and the uterine cannula was removed.

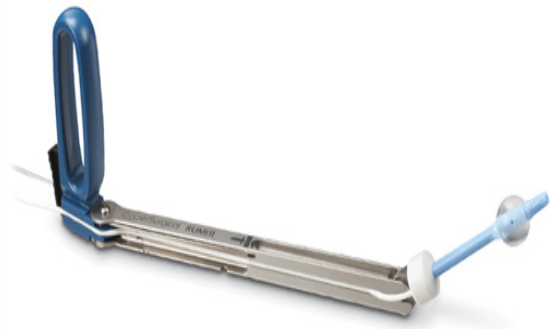

Fig 1: Uterine manipulator during the study.

Outcome: Tubal patency (patent or not) each tube is considered as one statistical unit

Statistical analysis: The recorded data were analyzed using the statistical package for social sciences, version 22 (SPSS Inc., Chicago, Illinois, USA). As mean \pm standard deviation (SD) quantitative data were expressed. Qualitative data were expressed in percentage and frequency.

The following tests were carried out: Chi-square $\left(\mathrm{x}^{2}\right)$ significance test was used to compare the proportions
Obstetrics and Gynecology 
between qualitative parameters. Kappa measure of agreement between laparoscopy and hysteroscopy in detecting Patent and Occluded: Slight agreement indicates 0-0.20; fair agreement; 0.21-0.40; moderate agreement; 0.41-0.60; substantial agreement 0.61 0.80 ; almost perfect agreement $0.81-1.00$. The interval of confidence was set at $95 \%$ and accepted error margin was set at $5 \%$. Consequently, the pvalue was considered as significant: Probability (Pvalue): P-value $\leq 0.05$ was deemed significant. Pvalue $\leq 0.001$ has been considered highly significant. $\mathrm{P}$-value $>0.05$ has been found insignificant.

\section{RESULTS}

This is a cross sectional cohort research conducted at the Department of Obstetrics and Gynecology at the University of Al Azhar (Bab El Shaaria and El Hussein University hospitals) within a period of 6 months. The protocol of this study was approved by the department \& college research ethics committees. The patients were counselled and written informed consent was obtained before inclusion in the study. One hundred infertile women referred for diagnostic/operative laparoscopy was included in the study.

A total of 100 women were recruited to the study during the period between January 2020 and June 2020. A total of 100 patients underwent diagnostic hysteroscopy followed by laparoscopy. Each fallopian tube has been considered as an independent case. Patency was evaluated in 200 Fallopian tubes.

\begin{tabular}{|c|c|}
\hline Demographic data & Total $(\mathbf{n}=\mathbf{1 0 0})$ \\
\hline Age (years) & \\
Range & $21-46$ \\
Mean \pm SD & $34.37 \pm 6.80$ \\
\hline BMI [wt/(ht) & \\
Range & $24-33$ \\
Mean \pm SD & $27.85 \pm 5.33$ \\
\hline
\end{tabular}

Table 1: Distribution of women according to their demographic data regarding age and BMI $(n=100)$. This table shows that the ranged age 21-46 with mean 34.37 \pm 6.80 , while ranged BMI 24-33 with mean $27.85 \pm 5.33$

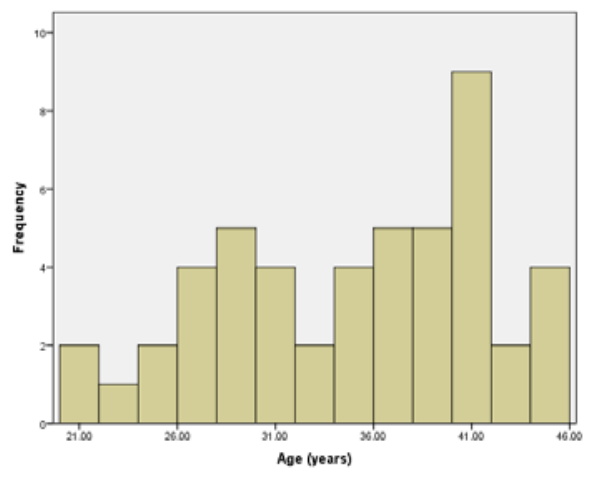

Fig 2: Box plot of women according to their demographic data regarding age.

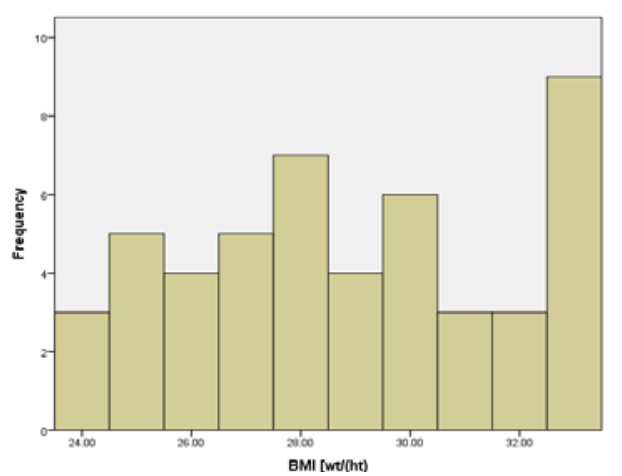

Fig 3: Box plot of women according to their demographic data regarding BMI.

\begin{tabular}{|c|c|c|}
\hline Gravidity & No. & \% \\
\hline Nulligravidas & 48 & $48.0 \%$ \\
\hline Gravida 1 & 22 & $22.0 \%$ \\
\hline Gravida 2 & 16 & $16.0 \%$ \\
\hline Gravida 3 & 8 & $8.0 \%$ \\
\hline Gravida 4 & 4 & $4.0 \%$ \\
\hline Gravida 5 & 2 & $2.0 \%$ \\
\hline Range [Mean \pm SD] & $0-5[1.02 \pm 1.22]$ \\
\hline
\end{tabular}

Table 2: Distribution of women according to their gravidity ( $\mathrm{n}=100)$.

This table shows that the Nulligravidas (48.0\%), Gravida 1 (22.0\%), Gravida 2 (16.0\%), Gravida 3 (8.0\%), Gravida 4 (4.0\%) and Gravida 5 (2.0\%) of Gravidity.

\begin{tabular}{|c|c|c|}
\hline Parity & No. & $\mathbf{\%}$ \\
\hline Nulliparas & 48 & $48.0 \%$ \\
\hline Para 1 & 30 & $30.0 \%$ \\
\hline Para 2 & 14 & $14.0 \%$ \\
\hline Para 3 & 8 & $8.0 \%$ \\
\hline Range [Mean \pm SD] & \multicolumn{2}{|c|}{$0-3[0.85 \pm 1.15]$} \\
\hline
\end{tabular}

Table 3: Distribution of women according to their parity $(\mathrm{n}=100)$.

This table shows that the Nulliparas (48.0\%), Para 1 (30.0\%), Para 2 (14.0\%), Para 3 (8.0\%) of Parity.

\begin{tabular}{|c|c|c|}
\hline Infertility & No. & $\mathbf{\%}$ \\
\hline Primary infertility & 55 & $55.0 \%$ \\
\hline Secondary infertility & 45 & $45.0 \%$ \\
\hline Duration of infertility (years) & \multicolumn{2}{|c|}{$1.5-16$} \\
Range [Mean \pm SD] & \multicolumn{2}{|c|}{$[4.27 \pm 3.87]$} \\
\hline
\end{tabular}

Table 4: Distribution of women according to their parity $(\mathrm{n}=100)$.

This table shows that the Primary infertility (48.0\%) and Secondary infertility (30.0\%) of infertility, while mean of duration of infertility $4.27 \pm 3.87$

\begin{tabular}{|c|c|c|}
\hline Hysteroscopy & No. & \% \\
\hline Patent on diagnostic hysteroscopy & 120 & $60 \%$ \\
\hline Occluded on diagnostic hysteroscopy & 80 & $40 \%$ \\
\hline
\end{tabular}

Table 5: Patency versus occlusion for individual fallopian tubes by diagnostic hysteroscopy $(n=200)$. 
This table shows that the Patent on diagnostic hysteroscopy (60\%) and Occluded on diagnostic hysteroscopy (40\%) by hysteroscopy.

The mean (SD) duration of the diagnostic hysteroscopy procedure was $196.12 \pm 63.37$ seconds ranged (85-355). Contralateral tubal orifices were equal in size in all patients. No dilatation or narrowing was observed in tubal orifices. A polyp was detected at one tubal orifice. Hyperemia was detected around one tubal orifice. Adhesions were detected at one tubal orifice.

\begin{tabular}{|c|c|c|}
\hline Laparoscopy & No. & $\mathbf{\%}$ \\
\hline Patent on diagnostic laparoscopy & 123 & $61.5 \%$ \\
\hline Occluded on diagnostic laparoscopy & 77 & $38.5 \%$ \\
\hline
\end{tabular}

Table 6: Patency versus occlusion for individual fallopian tubes by diagnostic laparoscopy $(n=200)$.

This table shows that the Patent on diagnostic hysteroscopy (61.5\%) and Occluded on diagnostic hysteroscopy (38.5\%) by hysteroscopy.

\begin{tabular}{|c|c|c|c|c|c|c|}
\hline \multirow{3}{*}{ 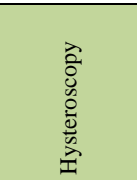 } & \multicolumn{4}{|c|}{ Laparoscopy } & \multirow{2}{*}{\multicolumn{2}{|c|}{ Kappa test }} \\
\hline & \multicolumn{2}{|c|}{$\begin{array}{l}\text { Patent on } \\
\text { laparoscopy }\end{array}$} & \multicolumn{2}{|c|}{$\begin{array}{l}\text { Occluded on } \\
\text { laparoscopy }\end{array}$} & & \\
\hline & No. & $\%$ & No. & $\%$ & $\mathrm{k}$ & $\begin{array}{c}\text { p- } \\
\text { value }\end{array}$ \\
\hline $\begin{array}{l}\text { Patent on } \\
\text { diagnostic }\end{array}$ & 113 & $91.9 \%$ & 7 & $9.1 \%$ & & \\
\hline $\begin{array}{l}\text { Occluded on } \\
\text { diagnostic }\end{array}$ & 10 & $8.1 \%$ & 70 & $\begin{array}{c}90.9 \\
\%\end{array}$ & 0.822 & $* *$ \\
\hline
\end{tabular}

${ }^{* *}$ p-value $<0.001 \mathrm{HS}$

Table 7: Agreement between laparoscopy and hysteroscopy in detection of tubal patency of the study group $(\mathrm{n}=200)$.

Statistical analysis of these results showed significant substantial agreement between the two modalities in the detection. Comparison of laparoscopy and hysteroscopy in detection of tubal patency a yielded weighted Kappa value of 0.822 , with p-value $<0.001$ HS

\begin{tabular}{|l|c|c|c|c|}
\hline \multirow{2}{*}{ Hysteroscopy } & \multicolumn{3}{|c|}{ Laparoscopy } \\
\cline { 2 - 5 } & $\begin{array}{c}\text { Patent on } \\
\text { laparoscopy }\end{array}$ & $\begin{array}{c}\text { Occluded on } \\
\text { laparoscopy }\end{array}$ \\
\cline { 2 - 5 } & No. & $\%$ & $\begin{array}{c}\text { N } \\
\text { o. }\end{array}$ & $\%$ \\
\hline Patent on diagnostic & 113 & $\begin{array}{c}91.9 \\
\%\end{array}$ & 7 & $9.1 \%$ \\
\hline $\begin{array}{l}\text { Occluded on } \\
\text { diagnostic }\end{array}$ & 10 & $\begin{array}{c}8.1 \\
\%\end{array}$ & 70 & $\begin{array}{c}90.9 \\
\%\end{array}$ \\
\hline $\begin{array}{l}\text { Sensitivity\% } \\
\text { 95\%C.I) }\end{array}$ & \multicolumn{3}{|c|}{$91.9 \%$ (85.5\%-97.4\%) } \\
\hline $\begin{array}{l}\text { Specificity\% } \\
\text { (95\%C.I) }\end{array}$ & \multicolumn{3}{|c|}{$90.9 \%$ (84.5\%-96.4\%) } \\
\hline $\begin{array}{l}\text { Positive predictive } \\
\text { value\% (95\%C.I) }\end{array}$ & \multicolumn{3}{|c|}{$94.2 \%$ (87.6\%-99.9\%) } \\
\hline $\begin{array}{l}\text { Negative predictive } \\
\text { value\% (95\%C.I) }\end{array}$ & \multicolumn{3}{|c|}{$87.5 \%$ (81.4\%-92.8\%) } \\
\hline Accuracy\% (95\%C.I) & \multicolumn{3}{|c|}{$91.5 \%$ (85.1\%-97.0\%) } \\
\hline p-value & \multicolumn{3}{|c|}{ (95.) } \\
\hline
\end{tabular}

Table 5: Diagnostic performance of Patency versus occlusion for individual fallopian tubes by diagnostic hysteroscopy and laparoscopy $(\mathrm{n}=200)$.
Bubble test during diagnostic hysteroscopy and chromo-pertubation during laparoscopy were used to assess the tubal patency. The tubes were patent in 113 cases for both methods. Occluded tubes were also diagnosed with laparoscopy and hysteroscopy in 70 cases. In 10 cases the tubes were patent by laparoscopic dye, but hysteroscopy could not be detected them as such. Occlusion was found by laparoscopy in 7 cases but patency was detected during the hysteroscopy. Of the non-patent cases, 70 out of 77 laparoscopic findings were consistent with the hysteroscopic result, yielding a specificity estimate of 90.9\% (84.5\%-96.4\%). In 113 out of 123 patent cases hysteroscopy was compatible with laparoscopy, achieving a sensitivity of $91.9 \%$ (85.5\%-97.4\%).

Positive and negative predictive values of $94.2 \%$ (87.6\%-99.9\%) and $87.5 \%$ (81.4\%-92.8\%), respectively were estimated. Hysteroscopic assessment had a 91.5\% (85.1\%-97.0\%) overall accuracy with the laparoscopic method taken as reference.

\section{DISCUSSION}

Fallopian tube obstruction plays a role in $12-33 \%$ of subfertile couples. Therefore, the assessment of the patency of fallopian tubes is a significant part of subfertile couple's work.$^{15}$ For this purpose, several tests are available including hysterosalpingography (HSG), laparoscopy and dye test, selective salpingography and hysterosalpingo-contrast sonography (HyCoSy). Each of these tests varies in inter and intra-observer reliability, diagnostic accuracy for predicting blockage or other tubal disease, prognostic information for independent pregnancy treatment, possible complications and costs ${ }^{6}$

Laparoscopy and dye test (also termed dye hydrotubation, dye insufflation, dye pertubation, chromopertubation or chromolaparoscopy) is widely considered a gold standard test for tubal patency investigation. It also allows peritubal disease assessments, adhesions and endometriosis. This has led to a recommendation by NICE (UK) that women suspected of having comorbidities (such as endometriosis and pelvic inflammatory disease) should undergo laparoscopy to assess pelvic and tubal pathology. Laparoscopy has the downside of being an invasive procedure. For this operation an operating room, anesthesia, and laboratory testing are mandatory. The cost of using the operating room, staff salaries, test costs and anaesthesiology consultation fees increase the total cost of the procedure. 6

Hysterosalpingography is a classic minimally invasive method for evaluation of tubal patency; however, hysterosalpingography is correlated with $72 \%$ to $85 \%$ sensitivity and $68 \%$ to $89 \%$ specificity in tubal patency diagnosis compared with laparoscopy. Moreover, HSG has drawbacks, including X-ray exposure, radio-contrast material and 
risk of infection. It's also an invasive procedure and relies on an expert radiologist. ${ }^{2}$

Positive ultrasound-enhancing contrast medium is used for hysterosalpingo-contrast sonography, with transvaginal ultrasound, to assess fallopian tube status and uterine cavity. The treatment can be carried out at the gynecologists' office. Air and saline solution with or without Doppler sonography can be used as a contrast agent; nonetheless, use of a dedicated contrast medium for ultrasound is generally recommended. This contrast medium is costly, which greatly increases the cost of the examination .10 The ultrasound saline contrast method used to assess the tubal status compared to the laparoscopic findings of chromotubations displayed $100 \%$ sensitivity and negative predictive value (NPV), but also 66\% low specificity and 57\% positive predictive value (PPV). The Echovist 's study of tubal patency yielded a better specificity (77\%) and PPV (70\%) (Radić et al., 2005). A metaanalysis validated that saline HyCoSy procedure had 76.1\% sensitivity and $79.4 \%$ specificity, $71.4 \%$ PPV and $83.1 \%$ NPV. HyCoSy and laparoscopy and the dye test findings were the same for 89 tubes, with a compliance rate of $78.1 \%$ indicating that Echovist had a $10.3 \%$ false positive rate for tubal occlusion diagnosis and a $6.7 \%$ false negative rate.$^{8}$

New techniques for assessing tubal patency in hysteroscopy have recently been described. The idea was to create a method for inspecting the tubes with as much accuracy as with laparoscopy, but to do so for a patient with less intrusion, less expense and less physical stress. Diagnostic hysteroscopy is an ideal diagnostic test because it is simple, easy, minimalinvasive, convenient, relatively cheap, fast, safe, accurate and widely used for every day practice by infertility-interested gynecologists.

Török and Major conducted selective perturbation with diagnostic hysteroscopy as part of an infertility evaluation prior to scheduled laparoscopy and chromohydrotubation in 35 infertile patients. During diagnostic hysteroscopy examination, a fine catheter inserted into the tubal ostea was described, following a blue methylene dye injection. If there was no reflux, this means the ostium was patent. Hysteroscopic tubal assessment was $82.9 \%$ accurate with the laparoscopic dye method used as a reference, 87.5 \% PPV and 76.7 $\%$ NPV. There was no complication or failure. They concluded that, the first line approach for uterine cavity and tubes evaluation in infertile women should be selective pertubation with diagnostic hysteroscopy. We do not think that selective perturbation with diagnostic hysteroscopy is ideal technique for assessment of tubal patency. A 5.5-mm operative bridge should be used and that would definitely cause intolerable pain. Intravasation or false passage due to unintentional perforation may occur because tubal cannulation occurs blindly without laparoscopic or sonographic surveillance. $^{16}$

Gynecologists recognize that the distention media using in diagnostic hysteroscopy often passes through patent fallopian tubes in to the pelvis.
Several authors have used the sonographic identification of new cul de sac (CDS) fluid as a patency proxy after diagnostic hysteroscopy. Limitations to this method include impeded evaluation when there is substantial pre-existing CDS fluid and difficulties in distinguishing between unilateral and bilateral patency ${ }^{1,17}$ Allamand coworkers compared the accuracy of HSG with diagnostic hysteroscopy combined with transvaginal ultrasound (TVS) in the diagnosis of tubal patency. The total saline usage for each hysteroscopic procedure at a fluid delivery system pressure of 100 $\mathrm{cm} \mathrm{H2O} \mathrm{was} 200 \mathrm{ml}$. The hysteroscope was withdrawn; transvaginal probe was reinserted in the sagittal plane to measure the free fluid in the cul-desac. In cul-de - sac, the volume of free fluid was measured in milliliters. The next day, HSG was done. The difference in tubal patency diagnosis between the two methods was compared using laparoscopy/chromotubation as a gold standard. According to the laparoscopy, $94.6 \%$ and $100 \%$ vs. $92.8 \%$ and $50 \%$ respectively were the sensitivity and specificity of hysteroscopy and HSG in detecting tubal patency. The best fluid volume cut off point in the cul-de -sac where both tubes are patent is $6 \mathrm{ml}$.

During diagnostic hysteroscopy, saline directly passing through the ostia may be difficult to observe unless there is a non-translucent substance, such as mucus and blood, which contrasts with saline. Many gynecologists have seen air mix unintentionally with their media of distention, spread through the ostia, and wonder if this really represents tubal patentcy. Air and saline are intentionally mixed with sonosalpingography for the assessment of tubal patency. If one envisioned a sonosalpingography when doing a hysteroscope (as if at the tip of the sonosalpingography catheter there was a camera), one would see air bubbles dispersing in the patency setting through the ostia, while staying in occlusion cavity.

The hypothesis of this study was to determine whether diagnostic hysteroscopy can accurately predict tubal patency. In the current study, diagnostic hysteroscopic bubble test has $91.9 \%$ sensitivity and $90.9 \%$ specificity in detection of tubal patency. The results of our study are in agreement with two previous studies which evaluated the accuracy of diagnostic hysteroscopic bubble test in detection of tubal patency .12,3 Parry conducted a prospective study to determine if air bubbles filled with saline during flexible diagnostic hysteroscopy will accurately predict tubal patency. The authors reported that the hysteroscopic approach has $98.3 \%$ sensitivity and $83.7 \%$ specificity in detection of tubal patency. ${ }^{12}$

A prospective study including 85 infertile patients compared the diagnostic accuracy of diagnostic hysteroscopy bubble test with HSG and laparoscopy. In 78 cases $(91.7 \%)$ the diagnostic hysteroscopic bubble test was feasible. Patent tubes were diagnosed with diagnostic hysteroscopy, HSG, and laparoscopy on both the right and left sides in 91 and 88.5, 92.3 and 91, and 93.6 and $93.6 \%$ respectively. The 
percentage of diagnostic hysteroscopy and laparoscopy agreements was $78 \%$, while tubal patency testing was $84 \%$ between HSG and laparoscopy. Diagnostic indices of diagnostic hysteroscopy were very close to those of HSG. The addition of diagnostic hysteroscopy to HSG did not improve accuracy in the diagnosis . ${ }^{3}$

\section{CONCLUSION}

Based on statistical analysis (sensitivity and specificity) of our data, diagnostic hysteroscopic bubble test is accurate and reliable compared to the laparoscopic test, which is considered a standard criterion worldwide. Diagnostic hysteroscopic is an efficient, precise, minimally invasive method for assessing tubal patency. It can be conducted as an inspection method that is as reliable, accurate, and repeatable as laparoscopy because of its low cost and minimal patient stress.

\section{REFERENCES}

1. Allam IS, Rashed AM, Sweedan KH, El Bishry GA, Ahmed WE (2014): Role of hysteroscopy in the evaluation of tubal patency in infertile women. Middle East Fertility Society Journal; 19(3), 21520.

2. Broeze KA, Opmeer BC, Van Geloven N, Coppus SF, Collins Ja, Den Hartog JE, et al. (2011): Are patients characteristics associated with the accuracy of Hysterosalpingography in giagnosing tubal pathology? An individual patient data meta analysis. Hum Reprod Update. 17:293-300.

3. Darwish AM, Hassanin AI, Aleem MA, Aboushama IH, Mohammad II (2016): A novel use of vaginoscopic diagnostic hysteroscopy for prediction of tubal patency and peristalsis among infertile women: a preliminary study. Gynecological Surgery; 13(3): 187-92.

4. Evangelista A, Oliveira MA, Crispi CP, Lamblet MF, Raymundo TS, Santos LC (2011): Diagnostic hysteroscopy using liquid distention medium: comparison of pain with warmed saline solution vs room-temperature saline solution. Journal of minimally invasive gynecology. 18(1):104-7.

5. Fouda UM, Gad Allah SH, Elshaer HS (2016): Optimal timing of misoprostol administration in nulliparous women undergoing diagnostic hysteroscopy: a randomized double-blind placebocontrolled study. Fertil Steril; 106(1): 196-201.

6. García LM, McFarlane E, Barnes S, Sanabria AJ, Alonso-Coello P, Alderson P (2014): Updated recommendations: an assessment of NICE clinical guidelines. Implementation Science. 9(1):72.
7. Garcia-Smith D and Effken JA (2013): Development and initial evaluation of the clinical information systems success model (CISSM). International Journal of Medical Informatics. 82(6):539-52.

8. Hamed HO, Shahin AY, Elsamman AM (2009): Hysterosalpingo-contrast sonography versus radiographic hysterosalpingography in the evaluation of tubal patency. Int J Gynaecol Obstet; 105: 215-7.

9. Lorincz J and Torok P (2019): Hysteroscopic assessment of fallopian tubal patency by air bubble sign. European Journal of Obstetrics and Gynecology and Reproductive Biology, 234: e121.

10. Ludwin I, Ludwin A, Wiechec M, Nocun A, Banas T, Basta P, Pitynski K (2017): Accuracy of hysterosalpingo-foam sonography in comparison to hysterosalpingo-contrast sonography with air/saline and to laparoscopy with dye. Human Reproduction. 32(4):758-69.

11. Mohammadifard M, Saburi A (2014): The role of other imaging modalities in evaluating the tubal patency. Journal of human reproductive sciences; 7(2): 154

12. Parry JP, Riche D, Aldred J, Isaacs J, Lutz E, Butler $\mathrm{V}$, et al. Proximal Tubal Patency Demonstrated Through Air Infusion During Flexible Diagnostic Hysteroscopy Is Predictive of Whole Tubal Patency. J Minim Invasive Gynecol. 2017; 4650(17)30138-43.

13. Phillips $\mathrm{CH}$, Benson CB, Ginsburg ES, Frates MC (2015): Comparison of uterine and tubal pathology identified by transvaginal sonography, hysterosalpingography, and hysteroscopy in female patients with infertility. Fertility Research and Practice; 1(1): 20.

14. Radić V, Čanić T, Valetić J, Duić Ž (2005): Advantages and disadvantages of hysterosonosalpingography in the assessment of the reproductive status of uterine cavity and fallopian tubes. European journal of radiology. 53(2):268-73.

15. Scholten I and Mol BW (2016): Defining infertility: global views on timing of IVF and the ability to access care. Fertility and Assisted Reproductive Technology (ART): Theory, Research, Policy and Practice for Health Care Practitioners. 235.

16. Török P and Major T (2012): Accuracy of assessment of tubal patency with selective pertubation at office hysteroscopy compared with laparoscopy in infertile women. Journal of Minimally Invasive Gynecology. 19(5):627-30.

17. Yildizhan B, Durmusoglu F, Uygur M, Erenus M (2009): A new technique for the diagnosis of fallopian tube patency by using hysteroscopy with ultrasound compared with hysterosalpingography in infertile women. Arch Gynecol Obstet.4(280): 543-7. 